\title{
Prevalence of Autoimmune Disease in Patients with Eosinophilic Esophagitis: A Cross-sectional Study of Three Hospitals in Japan
}

\author{
Maki Ayaki ${ }^{1}$, Noriaki Manabe ${ }^{1}$, Minoru Fujita ${ }^{1}$, Ryo Katsumata ${ }^{1}$, Jun Nakamura ${ }^{1}$, \\ Tomoari Kamada ${ }^{2}$, Masayuki Murota ${ }^{3}$, Kazuhiko Inoue $^{4}$ and Ken Haruma ${ }^{5}$
}

\begin{abstract}
:
Objective Recent studies have found higher rates of autoimmune diseases, such as celiac disease, Crohn's disease, and ulcerative colitis, in patients with eosinophilic esophagitis (EoE) than in the general population. This study investigated the concomitant rate of autoimmune disease among Japanese patients with $\mathrm{EoE}$ and evaluated the clinicopathological characteristics of EoE patients with autoimmune disease.

Methods The medical records of patients diagnosed with EoE between April 1, 2016, and June 30, 2020, were reviewed. We analyzed the concomitant rate of autoimmune diseases in patients with EoE and compared the clinical and histological differences between patients with and without autoimmune disease.

Results Sixty-four patients with EoE were enrolled. Of them, 1 had ulcerative colitis (1.6\%), 1 had autoimmune polyendocrine syndrome type 2 (1.6\%), and 6 had endometriosis $(28.5 \%$ of women, $44.4 \%$ of reproductive-aged women). No significant differences in the clinical course or histological findings were found between EoE patients with and without autoimmune diseases. No complications, including stenosis, were seen in EoE with autoimmune disease, and most patients with EoE and autoimmune diseases were responsive to proton pump inhibitors (PPIs).

Conclusion In this study, 8 out of 64 patients with EoE had an autoimmune-related disease, including ulcerative colitis $(n=1,1.6 \%)$, autoimmune polyendocrine syndrome type $2(n=1,1.6 \%)$, or endometriosis $(n=6$, $44.4 \%$ of women of reproductive age), which is higher than that previously reported in the general population. Further investigations are required to clarify the relationship between EoE and autoimmune diseases.
\end{abstract}

Key words: eosinophilic esophagitis, autoimmunity, regulatory $\mathrm{T}$ cells, autoimmune polyendocrine syndrome, ulcerative colitis, endometriosis

(Intern Med 60: 3525-3531, 2021)

(DOI: 10.2169/internalmedicine.7389-21)

\section{Introduction}

Eosinophilic esophagitis (EoE) is a clinicopathologic disease characterized by esophageal dysfunction and eosinophil-predominant inflammation in the esophagus (1). Its pathogenesis is incompletely understood; however, an association with autoimmune diseases, including Hashimoto's thyroiditis, celiac disease, Crohn's disease, ulcerative colitis
(UC), and rheumatoid arthritis, was identified in a Utah population-based cohort study (2). Another study reported that children with EoE had higher rates of type 1 diabetes (T1DM) and adrenal insufficiency than those without EoE (3). Furthermore, a recent genome-wide association study identified four new loci associated with EoE, two of which (c11orf30 and STAT6) were previously reported to have an association with autoimmune disease (4).

Thus, increasing numbers of reports have suggested a

\footnotetext{
${ }^{1}$ Division of Endoscopy and Ultrasonography, Department of Clinical Pathology and Laboratory Medicine, Kawasaki Medical School, Japan, ${ }^{2}$ Department of Health Care Medicine, Kawasaki Medical School, Japan, ${ }^{3}$ Department of Internal Medicine, Sakaide City Hospital, Japan, ${ }^{4}$ Junpukai Long Life Hospital, Japan and ${ }^{5}$ Division of Gastroenterology, Department of Internal Medicine 2, Kawasaki Medical School, Japan Received: February 20, 2021; Accepted: April 7, 2021; Advance Publication by J-STAGE: May 22, 2021

Correspondence to Dr. Maki Ayaki, mkmnb1979@yahoo.co.jp
} 
strong association between the disease processes of autoimmune disorders and EoE. However, many reports thus far on this association have been from Western countries, with few coming from Asian countries, including Japan.

The present study assessed the concomitant rate of autoimmune disease in Japanese patients with EoE and evaluated the differences in the clinicopathological characteristics of EoE patients with and without autoimmune disease.

\section{Materials and Methods}

\section{Patients and data collection}

The medical records of patients diagnosed with EoE in three Japanese clinical institutes between April 1, 2016, and June 30, 2020, were reviewed. The clinical institutes included one university hospital, one general hospital and one cancer screening center in Japan. A diagnosis of EoE in the present study was defined based on a biopsy finding of esophageal mucosal eosinophilia of $\geq 15$ eosinophils per high-power field (HPF) with symptoms of esophageal dysfunction or characteristic endoscopic findings of EoE, in accordance with the method used in our previous study (5). Patients whose biopsy findings showed esophageal mucosal eosinophilia caused by systemic factors, including eosinophilic gastroenteritis, parasites, drug hypersensitivity, and hyper-eosinophilic syndrome, were excluded. Patients who were younger than 16 years old or who had received acidsuppressive drugs [e.g., potassium-competitive acid blocker (P-CAB), proton-pump inhibitors (PPIs)] or steroid therapy were excluded from the study.

Information was collected from the medical records of these patients, including their sex, age and symptoms at presentation, as well as their clinical course and concomitant disease. Autoimmune diseases were defined in accordance with the lists of the American Autoimmune Related Diseases Association (AARDA) website (6). All concomitant diseases were collated according to the AARDA list, and then patients were divided into two groups: those with any diseases listed in the AARDA were classified into the autoimmune disease group, and those without any listed diseases were classified into the no-autoimmune disease group. Differences in clinicopathological characteristics (endoscopic findings, histological findings, and clinical course) were assessed between EoE patients with and without autoimmune disease.

\section{The evaluation of endoscopic findings}

Endoscopic findings related to EoE (e.g., edema, rings, exudates, furrows, and strictures) based on the EoE endoscopic reference score (EREFS) (7) were reviewed in random order (for blinding) by three of the authors who were blinded to patients' information. Discrepancies were resolved by consensus. The total score of the 5 major EREFS findings was calculated and categorized on a 10-point scale from 0 to 10 in accordance with our previous study (5).

\section{The evaluation of histological findings and interob- server agreement}

The number of eosinophils was counted and scored based on the peak eosinophil count (PEC) within an HPF $(1=<15$ eosinophils/HPF; 2=15-59 eosinophils/HPF; $3=\geq 60$ eosinophils/HPF). Basal zone hyperplasia (BZH) and dilated intercellular spaces (DIS) were also reviewed, and each feature was scored from 0-3 points in accordance with the histology scoring system (EoEHSS) (8). If the biopsy specimens were horizontally sectioned and did not contain whole mucosal epithelium from the superficial to basal layers with basal membrane, a BZH score was not calculated.

After one of the authors (M.A.) had explained how to score PEC, BZH, and DIS using several representative histological images to two randomly selected authors, a histological evaluation was independently performed by these authors, without access to any patient's information. Discrepancies were resolved by consensus. To determine the reliability of the endoscopic and histological evaluations, interobserver agreement was assessed among these three authors. In addition, interobserver agreement was evaluated between one of the authors (M.A.) and one of the expert pathologists in our institution who was not an author of this paper.

\section{Statistical analyses}

Data are expressed as the mean \pm standard deviation. Student's $t$-test was used to compare the mean values of two independent groups. To compare categorical data, we used the chi-squared test with Yates' correction or Fisher's exact test. $P$ values $<0.05$ were considered to indicate statistical significance. Kappa statistics with $95 \%$ confidence intervals (CIs) were calculated in accordance with Cohen's kappa calculation. Kappa values were evaluated as follows: $\leq 0.20$, poor; 0.21-0.40, fair; 0.41-0.60, moderate; 0.61-0.80, substantial; and 0.81-1.00, almost perfect.

All statistical analyses were performed using the SPSS Statistics software program for Windows, version 22.0 (IBM, Armonk, USA).

\section{Ethical considerations}

The study was performed according to the ethical guidelines of the 1975 Declaration of Helsinki and was approved by our Institutional Review Board. Written informed consent was obtained from each patient included in the study.

\section{Results}

Seventy-two patients were diagnosed with EoE during the study period. Six patients were excluded because they had already received a PPI. No patient received systematic or topical steroid therapy. Two patients (a 7-year-old girl and an 8-year-old boy) were excluded because their age did not meet the inclusion criteria. Ultimately, 64 patients were included in the analysis. 


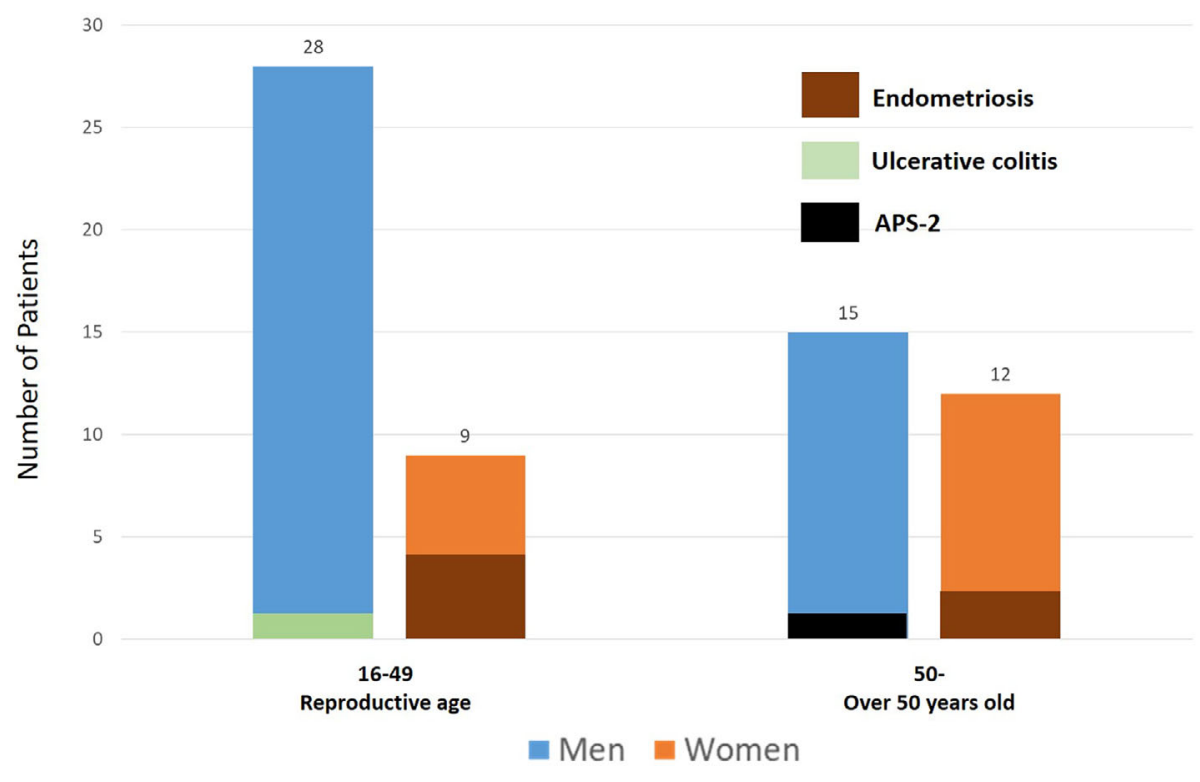

Figure 1. Sex and number of patients with eosinophilic esophagitis and autoimmune diseases in reproductive and non-reproductive age groups (over 50 years old). The predominance of men was more pronounced in patients with eosinophilic esophagitis of reproductive age group than in the nonreproductive age group. The rate of autoimmune disease coexistence was higher in women than in men.

\section{Patient characteristics}

The 64 enrolled patients comprised 43 men and 21 women, with a mean age of 47.1 13.2 years (range 16-79). According to the World Health Organization (WHO) classification (9), there were 37 patients of reproductive age (1649 years old) (28 men, 9 women) and 27 patients of nonreproductive age (over 50 years old) (15 men, 12 women). The proportion of reproductive-aged men was higher than that in the non-reproductive-age group, although the difference was not statistically significant $(\mathrm{p}=0.111)$ (Fig. 1). No patients had a family history of EoE. Sixteen patients $(25 \%)$ had peripheral blood eosinophilia ( $\geq 500$ eosinophils $/ \mu \mathrm{L}$ ), but none had gastric or duodenal eosinophilia. We analyzed the serum IgE levels from 42 of the 64 patients with EoE, and $30(71.4 \%)$ had elevated total $\mathrm{IgE}$ levels.

\section{Concomitant disease}

Allergic disease was detected in 40 patients $(62.5 \%)$, as follows: allergic rhinitis $(n=19,29.7 \%)$, bronchial asthma ( $n$ $=10,15.6 \%)$, atopic dermatitis $(n=7,10.9 \%)$, food allergy $(n$ $=6,9.4 \%)$, drug allergy $(n=1)$, and metal allergy $(n=1)$. Of note, some cases had more than one allergic disease.

Comorbid diseases other than allergic diseases were as follows: 11 patients had hypercholesterolemia (17.1\%), 6 patients had hypertension (9.4\%), 6 patients had endometriosis $(28.5 \%$ of women, $44.4 \%$ of reproductive-aged women), 3 patients had non-alcoholic steatohepatitis (NASH) (4.7\%), 2 patients had polycystic ovarian syndrome (PCOS) $(9.5 \%$ of women, $22.2 \%$ of reproductive-aged women), 2 patients had gastric cancer $(3.1 \%), 1$ patient had ovarian teratoma (4.8\% of women), 1 patient had breast cancer $(4.8 \%$ of women), 1 patient had autoimmune polyendocrine syndrome type 2 (APS-2) (1.6\%), 1 patient had UC (1.6\%), 1 patient had palmoplantar pustulosis(1.6\%), and 1 patient had HIV infection $(1.6 \%)$.

\section{Autoimmune disease and EoE}

According to the AARDA list (6), UC, APS-2, and endometriosis are classified as autoimmune diseases.

\section{- UC}

The prevalence of UC in EoE was 1/64 (1.6\%). The patient (an 18-year-old man) was in remission by biologic anti-tumor necrosis factor- $\alpha$ (TNF) therapy. Four years prior to the diagnosis of EoE, he had undergone esophagogastroduodenoscopy (EGD), the findings of which were normal. EoE was diagnosed based on the presence of dysphagia and characteristic endoscopic findings, and a PPI was effective.

\section{- APS-2}

One patient (a 67-year-old man) had APS-2, including Hashimoto's thyroiditis, T1DM, Sjögren's syndrome, and autoimmune gastritis (10). The prevalence of APS-2 in EoE was $1 / 64(1.6 \%)$, with the same values for Hashimoto's thyroiditis, T1DM, Sjögren's syndrome, and autoimmune gastritis. Two years prior to the diagnosis of EoE, he had undergone EGD for health screening and been diagnosed only with atrophic gastritis. He did not respond to PPIs but rapidly improved symptomatically and endoscopically with topical steroid therapy (10).

\section{- Endometriosis}

Six women $(25,26,41,49,53$, and 55 years old) had a history of endometriosis with a prevalence of $6 / 21(28.6 \%$ of all women) and 4/9 (44.4\% of women of reproductive age) (Fig. 1). Five of these six patients responded well to 


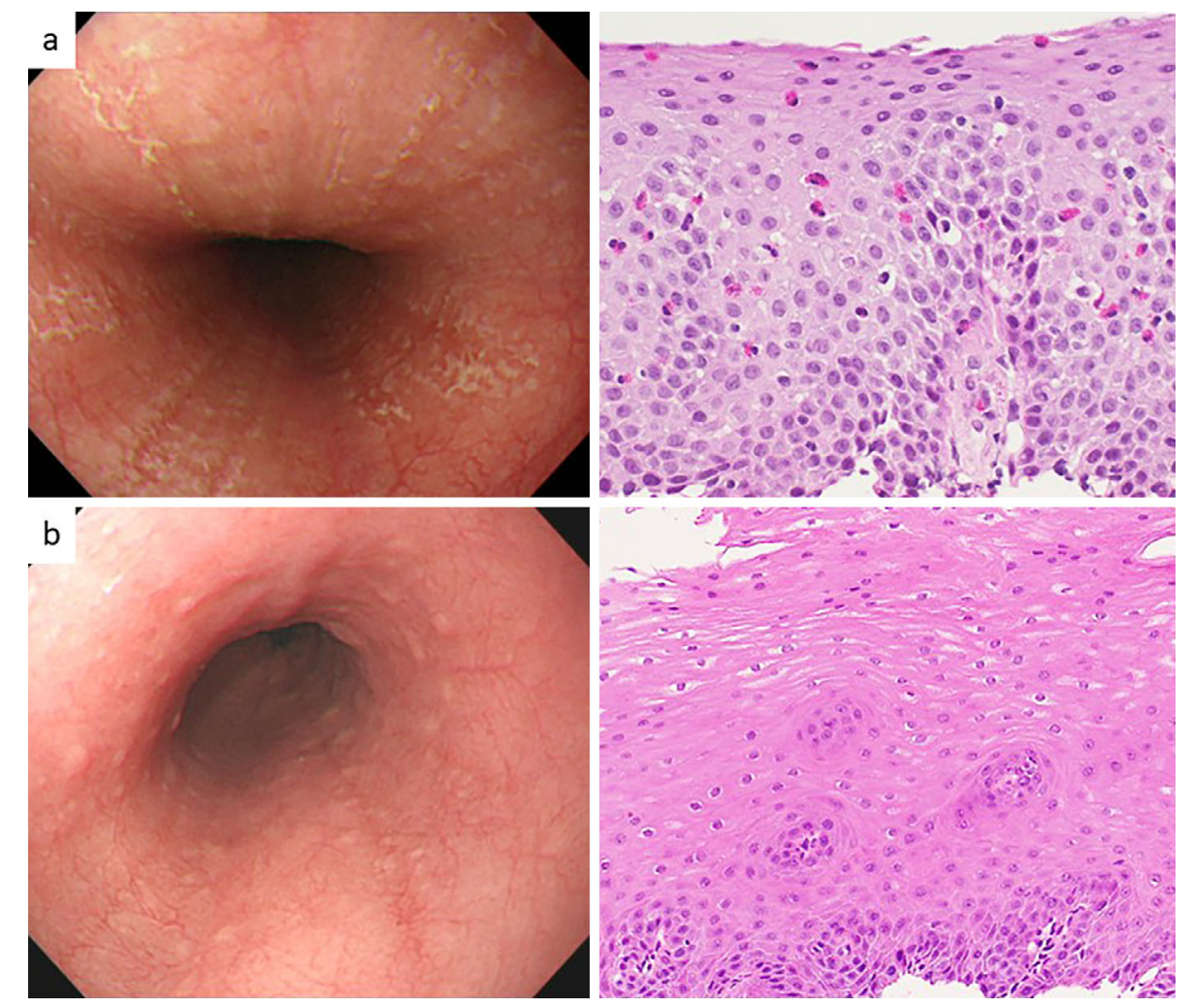

Figure 2. Endoscopic and histological images of a patient in remission with proton pump inhibitor (PPI) therapy. (a) At the diagnosis. Longitudinal exudates are present. Histological findings in a biopsy specimen from the exudate indicate eosinophilic infiltration. (b) After 12 weeks of PPI therapy. Normal mucosa is present. A histological analysis shows that the eosinophilic infiltration has disappeared.

PPI therapy (Fig. 2). One patient had minimal symptoms and endoscopic findings but did not respond to PPIs. The symptoms were very mild and did not require a change in treatment.

\section{Differences in the clinicopathological characteristics of patients with and without autoimmune diseases}

Regarding endoscopic findings, total EREFS scores were not significantly different between EoE patients with and without autoimmune disease $(3.00 \pm 0.45$ vs. $3.00 \pm 0.89)$. The incidence of each endoscopic finding in the groups with and without autoimmune disease was as follows: edema $(100.0 \%$ vs. $98.2 \%, \mathrm{p}=1.00)$, rings $(37.5 \%$ vs. $42.9 \%, \mathrm{p}=1.00)$, exudates $(37.5 \%$ vs. $53.6 \%, \mathrm{p}=1.00)$, furrows $(87.5 \%$ vs. $96.4 \%$, $\mathrm{p}=0.335)$, and stricture $(0.0 \%$ vs. $3.6 \%, \mathrm{p}=1.00)$.

Furthermore, there were no significant differences in histopathological finding scores between EoE patients with and without autoimmune disease: PEC $(2.40 \pm 0.40$ vs. $2.29 \pm$ $0.49, \mathrm{p}=0.605), \mathrm{BZH}(2.25 \pm 0.43$ vs. $2.31 \pm 0.39, \mathrm{p}=0.863)$, and DIS (2.00 \pm 0.54 vs. $2.65 \pm 0.12, \mathrm{p}=0.107)$ (Table).

\section{Interobserver agreement for the evaluation of histo- pathological finding scores}

The kappa value of interobserver agreement for the evaluation of the histopathological finding scores was $0.80(95 \%$ CI: 0.43-1.17) among the 3 evaluators and 0.80 (95\% CI:
0.43-1.17) between 1 author (M.A.) and 1 expert pathologist who was not an author of this paper, thereby indicating substantial diagnostic agreement.

There were no substantial differences in responses to treatments. Eighty-one percent of EoE patients without autoimmune diseases responded to a PPI or P-CAB, and most of them had a good disease course, although two cases developed severe stenosis.

\section{Discussion}

This study examining the overlap between EoE and autoimmune disease in Japan had several notable findings. First, among the 64 patients with EoE, 1 (1.6\%) each had UC and APS-2. The prevalence rate of UC reported in Japan was $0.17 \%$ (11), the prevalence of APS-2 in Japan is unknown, and the prevalence of T1DM, a major component of APS-2, was reported to be $0.1 \%$ (12).

Kappelman et al. (13) reported a higher prevalence rate of UC in EoE patients than in the US general population $(0.8 \%$ vs. $0.4 \%$ ). The prevalence of T1DM in EoE patients was $1.2 \%$, which was 4 times higher than the prevalence in the general population in a large US cohort of children (3). The prevalences of UC and T1DM in EoE patients in this study were similar to those reported from the US.

Another notable finding in our study was that the preva- 
Table. Clinical Differences with and without Coexistence of Autoimmune Diseases.

\begin{tabular}{|c|c|c|c|}
\hline & $\begin{array}{l}\text { With-autoimmune } \\
\text { disease patients }(n=8)\end{array}$ & $\begin{array}{l}\text { Without-autoimmune } \\
\text { disease patients }(n=56)\end{array}$ & $\mathrm{p}$ value \\
\hline Age $(y r)$, mean (range) & $41.4(17-61)$ & $47.5(16-79)$ & 0.36 \\
\hline Male, n (\%) & $2(25.0 \%)$ & $41(73.2 \%)$ & $0.012 *$ \\
\hline \multicolumn{4}{|l|}{ Symptoms (duplicate count) } \\
\hline None, $\mathrm{n}(\%)$ & 0 & $27(48.2 \%)$ & 0.062 \\
\hline Dysphagia, n (\%) & $6(75.0 \%)$ & $36(64.3 \%)$ & 1 \\
\hline Chest pain, $\mathrm{n}(\%)$ & $2(25.0 \%)$ & $1(1.8 \%)$ & $0.014 *$ \\
\hline Food impaction, $\mathrm{n}(\%)$ & 0 & $2(3.6 \%)$ & 1 \\
\hline \multicolumn{4}{|l|}{ Endoscopic findings } \\
\hline EREFS total score & $3.00 \pm 0.45$ & $3.00 \pm 0.89$ & 0.655 \\
\hline Edema $\geq$ Grade1, n (\%) & $8(100 \%)$ & $55(98.2 \%)$ & 1 \\
\hline Rings $\geq$ Grade $1, \mathrm{n}(\%)$ & $3(37.5 \%)$ & $24(42.9 \%)$ & 1 \\
\hline Exudates $\geq$ Grade1, n (\%) & $3(37.5 \%)$ & $30(53.6 \%)$ & 1 \\
\hline Furrows $\geq$ Grade1, n (\%) & $7(87.5 \%)$ & $54(96.4 \%)$ & 0.335 \\
\hline Stricture $\geq$ Grade $1, \mathrm{n}(\%)$ & 0 & $2(3.6 \%)$ & 1 \\
\hline \multicolumn{4}{|l|}{ Histological findings } \\
\hline PEC grade score, mean (SD) & $2.40(0.40)$ & $2.29(0.49)$ & 0.605 \\
\hline BZH grade score, mean (SD) & $2.25(0.43) \dagger$ & $2.31(0.39) \ddagger$ & 0.863 \\
\hline DIS grade score, mean (SD) & $2.00(0.54)$ & $2.65(0.12)$ & 0.107 \\
\hline
\end{tabular}

†One unevaluated case was excluded. $\$$ Eighteen unevaluated cases were excluded. P-values indicate statistical significance $(* \mathrm{p}<0.05)$.

EREFS: EoE Endoscopic Reference Score, PEC: peak eosinophil count, BZH: basal zone hyperplasia, DIS: dilated intercellular spaces

lence of endometriosis was extremely high in female EoE patients of reproductive age. To our knowledge, there is no report discussing the association between EoE and endometriosis. Endometriosis is a chronic inflammatory disease affecting 6-10\% of women of reproductive age and is associated with an altered immune response to endometrial cells, facilitating the implantation and proliferation of ectopic endometrial tissues (14). Recently, it was reported that the dysregulation of Tregs contributes to the growth of ectopic lesions in patients with endometriosis $(15,16)$. Statistically significant associations with endometriosis have been reported for systemic lupus erythematosus, Sjögren's syndrome, rheumatoid arthritis, celiac disease, and multiple sclerosis (17). Women with endometriosis have been reported to have four times the prevalence of allergic diseases (18) and a higher incidence of UC, Crohn's disease, and celiac disease than general population (19). Women of reproductive age who had EoE had more endometriosis than those in the general population, suggesting that sex hormones may play an important role in the development of EoE. Furthermore, among sex hormones, estrogen is an important regulator of the immune system, and marked changes in estrogen levels due to menopause and other factors can cause autoimmune diseases (20). Indeed, ovariectomy leads to estrogen deficiency, which exacerbated Sjögren's syndrome in a murine model (21). Xiong et al. showed that estrogen upregulated Tregs, which play an important role in immune tolerance (22). All of the autoimmune diseases found in the present study (endometriosis, APS-2, and UC) have been reported to be associated with
Tregs (23-25). Tregs play a role in maintaining peripheral self-tolerance as well as tolerance to harmless non-self antigens, such as those associated with oral food tolerance (26). Furthermore, a relative lack of Tregs was reported in adult patients with EoE (27). Thus, Treg dysfunction has been reported in patients with autoimmune disease and those with EoE. This common mechanism might contribute to the higher rates of autoimmune disease in EoE patients than in the general population, although further studies are necessary to confirm these findings.

Johnson et al. showed that the rate of potential or probable celiac disease in patients with EoE was $13.6 \%$ in a cohort of US adults (28). In contrast, we did not identify any patients with celiac disease in our cohort. In Western countries, the prevalence of celiac disease was reported to be as high as $1 \%$, whereas the prevalence in Japan was $0.05 \%$ (29). Patients carrying HLA-DQ2 or HLA-DQ8 are considered at high risk of developing celiac disease (30-32). Because the prevalence of HLA-DQ2 and HLA-DQ8 is very low in Japan, the number of Japanese patients with celiac disease is likely to be small, and this genetic background may have contributed to our finding that no patients had celiac disease.

In the present study, there were no significant differences in the endoscopic or histological findings based on the presence or absence of autoimmune disease. Fan et al. reported no substantial clinical, endoscopic, or histologic differences between EoE patients with and without inflammatory bowel disease (IBD) (33). In several case reports of coexisting Crohn's disease and EoE (34-36), EoE developed after the 
induction of remission of Crohn's disease, suggesting that these diseases might be reciprocal. EoE is a TH2 type disease, and Crohn's disease is a TH1 type disease (37), and coexisting TH1 and TH2 diseases have been shown to have reciprocal or additive effects (38). In cases of co-existing EoE and autoimmune diseases, physicians should be aware these diseases may interact with each other.

The limitations of our study included the small number of cases and its retrospective nature. However, the mean age, ratio of men to women, and percentage of previous allergic disease were consistent with previous reports of EoE from other institutions in Japan, and there was no bias (39). Second, not all patients were tested for autoantibodies or had seen a gynecologist; therefore, some patients might have had undiagnosed hypothyroidism or endometriosis. A larger, prospective, study of adult patients with EoE is needed to assess the prevalence of autoimmune disease more accurately.

In summary, Japanese patients with EoE might have a high concomitant rate of autoimmune disease, which is consistent with several previous reports from the US. However, the clinical course of EoE was not significantly different between the groups with and without autoimmune disease.

\section{Conclusion}

The findings of the present study support the close association between EoE and autoimmune disease. Further studies are needed to establish the contribution of autoimmunity in EoE.

The authors state that they have no Conflict of Interest (COI).

\section{Acknowledgement}

We thank J. Ludovic Croxford, PhD, for editing a draft of this manuscript.

\section{References}

1. Dellon ES. Epidemiology of eosinophilic esophagitis. Gastroenterol Clin North Am 43: 201-218, 2014.

2. Peterson K, Firszt R, Fang J, et al. Risk of autoimmunity in eoe and families, a population-based cohort study. Am J Gastroenterol 111: 926-932, 2016.

3. Capucilli P, Cianferoni A, Grundmeier RW, et al. Comparison of comorbid diagnoses in children with and without eosinophilic esophagitis in a large population. Ann Allergy Asthma Immunol 121: 711-716, 2018.

4. Sleiman PM, Wang ML, Cianferoni A, et al. GWAS identifies four novel eosinophilic esophagitis loci. Nat Commun 5: 5593, 2014.

5. Ayaki M, Manabe N, Nakamura J, et al. The "caterpillar sign": a novel endoscopic indicator of eosinophilic esophagitis. Esophagus 18: 156-162, 2021.

6. American Autoimmune Related Diseases Association [Internet]. [cited 2021 Apr 30]. Available from: http://www.aarda.org

7. Hirano I, Moy N, Heckman MG, et al. Endoscopic assessment of the oesophageal features of eosinophilic oesophagitis: validation of a novel classification and grading system. Gut 62: 489-495, 2013.

8. Collins MH, Martin LJ, Alexander ES, et al. Newly developed and validated eosinophilic esophagitis histology scoring system and evidence that it outperforms peak eosinophil count for disease di- agnosis and monitoring. Dis Esophagus 30: 1-8, 2017.

9. World Health Organization. Reproductive health indicators for global monitoring: report of the second interagency meeting, WHO Geneva, 17-19 July 2000. World Health Organization [Internet]. [cited 2021 Apr 30]. Available from: https://apps.who.int/iris/ handle/10665/66918

10. Ayaki M, Manabe N, Fujita M, et al. A case of eosinophilic esophagitis with autoimmune polyendocrine syndrome type 2, including autoimmune gastritis. Clin J Gastroenterol 14: 460-465, 2021.

11. Murakami Y, Nishiwaki Y, Oba MS, et al. Estimated prevalence of ulcerative colitis and Crohn's disease in Japan in 2014: an analysis of a nationwide survey. J Gastroenterol 54: 1070-1077, 2019.

12. Nishioka $Y$, Noda $T$, Okada $S$, et al. Incidence and seasonality of type 1 diabetes: a population-based 3-year cohort study using the National Database in Japan. BMJ Open Diabetes Res Care 8: e001262, 2020.

13. Kappelman MD, Rifas-Shiman SL, Kleinman K, et al. The prevalence and geographic distribution of Crohn's disease and ulcerative colitis in the United States. Clin Gastroenterol Hepatol 5: 14241429, 2007.

14. Giudice LC. Clinical practice. Endometriosis. N Engl J Med 362: 2389-2398, 2010.

15. Tanaka Y, Mori T, Ito F, et al. Exacerbation of endometriosis due to regulatory T-cell dysfunction. J Clin Endocrinol Metab 102: 3206-3217, 2017.

16. Deobandi Ali-Akbar, et al. Higher frequency of circulating, but not tissue regulatory $\mathrm{T}$ cells in patients with endometriosis. J Reprod Immunol 139: 103119, 2020.

17. Janssen OE, Mehlmauer N, Hahn S, et al. High prevalence of autoimmune thyroiditis in patients with polycystic ovary syndrome. Eur J Endocrinol 150: 363-369, 2004.

18. Matalliotakis I, Cakmak H, Matalliotakis M, Kappou D, Arici A. High rate of allergies among women with endometriosis. J Obstet Gynaecol 32: 291-293, 2012.

19. Jess T, Frisch M, Jørgensen KT, Pedersen BV, Nielsen NM. Increased risk of inflammatory bowel disease in women with endometriosis: a nationwide Danish cohort study. Gut 61: 12791283, 2012.

20. Nadkarni S, McArthur S. Oestrogen and immunomodulation: new mechanisms that impact on peripheral and central immunity. Curr Opin Pharmacol 13: 576-581, 2013.

21. Ishimaru N, Saegusa K, Yanagi K, et al. Estrogen deficiency accelerates autoimmune exocrinopathy in murine Sjögren's syndrome through Fas-mediated apoptosis. Am J Pathol 155: 173-181, 1999.

22. Xiong YH, Yuan Z, He L. Effects of estrogen on CD4+ CD25+ regulatory $\mathrm{T}$ cell in peripheral blood during pregnancy. Asian Pac J Trop Med 6: 748-752, 2013.

23. de Barros IB, Malvezzi H, Gueuvoghlanian-Silva BY, et al. "What do we know about regulatory $\mathrm{T}$ cells and endometriosis? A systematic review”. J Reprod Immunol 120: 48-55, 2017.

24. Kriegel MA, Lohmann T, Gabler C, et al. Defective suppressor function of human CD4+CD25+ regulatory $\mathrm{T}$ cells in autoimmune polyglandular syndrome type II. J Exp Med 199: 1285-1291, 2004.

25. Takahashi M, Nakamura K, Honda K, et al. An inverse correlation of human peripheral blood regulatory $\mathrm{T}$ cell frequency with the disease activity of ulcerative colitis. Dig Dis Sci 51: 677-686, 2006.

26. Sakaguchi S, Miyara M, Costantino CM, et al. FOXP3+ regulatory $\mathrm{T}$ cells in the human immune system. Nat Rev Immunol 10: 490500, 2010.

27. Stuck MC, Straumann A, Simon HU. Relative lack of T regulatory cells in adult eosinophilic esophagitis - no normalization after corticosteroid therapy. Allergy 66: 705-707, 2011. 
28. Johnson JB, Boynton KK, Peterson KA. Co-occurrence of eosinophilic esophagitis and potential/probable celiac disease in an adult cohort: a possible association with implications for clinical practice. Dis Esophagus 29: 977-982, 2016.

29. Fukunaga M, Ishimura N, Fukuyama C, et al. Celiac disease in non-clinical populations of Japan. J. Gastroenterol 53: 208-214, 2018.

30. Hadithi M, von Blomberg BM, Crusius JB, et al. Accuracy of serologic tests and HLA-DQ typing for diagnosing celiac disease. Ann Intern Med 147: 294-302, 2007.

31. Kim CY, Quarsten H, Bergseng E, et al. Structural basis for HLADQ2-mediated presentation of gluten epitopes in celiac disease. Proc Natl Acad Sci USA 101: 4175-4179, 2004.

32. Lundin KE, Gjertsen HA, Scott $H$, et al. Function of DQ2 and DQ 8 as HLA susceptibility molecules in celiac disease. Hum Immunol 41: 24-27, 1994.

33. Fan YC, Steele D, Kochar B, et al. Increased prevalence of esophageal eosinophilia in patients with inflammatory bowel disease. Inflamm Intest Dis 3: 180-186, 2019.

34. Mulder DJ, Hookey LC, Hurlbut DJ, et al. Impact of crohn disease on eosinophilic esophagitis: evidence for an altered TH1-TH2 immune response. J Pediatr Gastroenterol Nutr 53: 213-215, 2011.

35. Suttor VP, Chow C, Turner I. Eosinophilic esophagitis with Crohn's disease: a new association or overlapping immune - mediated enteropathy? Am J Gastroenterol 104: 794-795, 2009.

36. Miyaoka Y, Tsukano K, Ueno S, et al. A case of eosinophilic esophagitis complicated with Crohn's disease. Gastroenterol Endosc 57: 128-133, 2015.

37. MacDonald TT, Monteleone G. Overview of role of the immune system in the pathogenesis of inflammatory bowel disease. Adv Exp Med Biol 579: 98-107, 2006.

38. Tian J, Olcott AP, Hanssen LR, et al. Infectious Th1 and Th2 autoimmunity in diabetes-prone mice. Immunol Rev 164: 119-127, 1998.

39. Kitamura H, Tanaka F, Nadatani Y, et al. Eosinophilic esophagitis and asymptomatic esophageal eosinophilia display similar immunohistological profiles. J Clin Biochem Nutr 68: 246-252, 2021.

The Internal Medicine is an Open Access journal distributed under the Creative Commons Attribution-NonCommercial-NoDerivatives 4.0 International License. To view the details of this license, please visit (https://creativecommons.org/licenses/ by-nc-nd/4.0/).

(C) 2021 The Japanese Society of Internal Medicine

Intern Med 60: 3525-3531, 2021 